\title{
Overall Response
}

National Cancer Institute

\section{Source}

National Cancer Institute. Overall Response. NCI Thesaurus. Code C96613.

An assessment of the overall response of the disease to the therapy. 